\title{
BACTERIAL TRANSLOCATION OF PROBIOTIC LACTOBACILLI
}

\section{O. Aheyev}

The effectiveness of probiotic preparations based on lactic acid bacteria is caused by the ability of the latter to colonize the inner surface of the digestive tract and produce a number of biologically active metabolites (organic acids, primarily lactic, lysozymes, etc.), which not only helps protect the body against pathogens, but also activates its own protective and adaptive reaction. At the end of the twentieth century there appeared more experimental data that contradicts the traditional idea of the mechanisms of impact of probiotics on a microorganism. The authors from different countries pointed out the cases of efficacy of probiotic preparations that are not subject to explanation in terms of conventional vision not only to treat infections of the digestive tract in the conditions of the intestine colonization, but also for the treatment of pathological processes localized outside the gastrointestinal tract [1-4].

In this regard, of particular importance is the study of the phenomenon of translocation of probiotic strains of microorganisms of different taxonomic groups (bacilli, Escherichia coli, bifidobacteria, lactobacilli, etc.), which are the basis of veterinary and medical preparations of different functional orientation (preventive, therapeutic, stimulating), its dynamics, clarifying the response of the microorganism on indicators of morphological changes in organs and its main systems activity. This allows significantly expand the understanding of the complex process of interaction of the probiotics with a macroorganism.

Today it is urgent to develop new approaches to the strategy and tactics for the development and use of bacterial biological products, including probiotics. And thanks to a comprehensive study of mutual influence of probiotic bacteria and macroorganism it becomes possible to create probiotic preparations intended for the prevention and treatment of disorders not only of the digestive system, but many diseases of farm animals and humans that are located outside the gastrointestinal tract [5-7] .

One of the mechanisms of such an action of probiotics is their stimulation of nonspecific resistance of macroorganism as a result of translocation of bacteria not only of the upper gastrointestinal tract (oropharynx and stomach) - similar processes also continue during further passage of microorganisms through all parts of the gastrointestinal tract. In addition, biologically active compounds produced by cells of the intestine in response to interactions with bacteria and are produced directly by microorganisms make an important effect on the stimulation of the immune system, by being soaked in blood and lymph [8].

Given the above, the objective of the work was to determine the dependence of bacterial translocation on the strain of probiotic lactic acid bacteria.

Materials and methods. The strains of lactic acid bacteria Lactobacillus plantarum L5 (part of the preparation БПС-Л) [9] and $L$. plantarum Lc-18 from the collection of probiotics laboratory, promising to create a new bacterial drug on its basis, were used for the research. Growing the cultures of lactic acid bacteria was carried out on de Man nutrient medium (MRS) [10]. Growing test cultures to determine the antagonistic activity was carried out on the surface of meat-and-peptone agar (MPA) [11].

Before the study of bacterial translocation cultural-morphological (the morphology of cells and colonies, especially the growth on nutrient media) and physiological and biochemical (the activity of ectoenzymes, the ability to ferment various sources of carbon, antagonistic activity) properties of the original probiotic cultures were determined, that distinguish them from other lactic acid bacteria representatives of normal microflora of animals [11-14].

To study the translocation dynamics of investigated bacteria in the blood of animals there were formed 5 groups of laboratory rabbits numbered 3 heads each - one control and four experimental; control animals were intact (received the appropriate amount of saline), animals of experimental group-1 were given the culture of the bacteria strain Lactobacillus plantarum L5 at the dose of $3 * 10^{8} \mathrm{CFU}$, animals of experimental group- 2 - the same 
strain at the dose of $3^{*} 10^{9} \mathrm{CFU}$, experimental group-3 - the culture of $L$. plantarum Lc-18 at the dose of $3 * 10^{8} \mathrm{CFU}$, experimental group- 4 the same culture at the dose of $3 * 10^{9} \mathrm{CFU}$. The suspensions of bacterial cells in saline were injected to animals once orally. Within 3,10 , 30, 60 min., $24 \mathrm{hr}$., 7 and 14 days after the administration of bacteria in each animal blood samples were taken.

To study the translocation dynamics of investigated bacteria in parenchymal organs (liver, spleen, kidneys) of laboratory animals there were formed 5 groups of white mice numbered 15 heads each - one control and four experimental; control animals were intact (received the appropriate amount of saline), animals of experimental group-1 were given the culture of the bacteria strain L. plantarum L5 at the dose of $3 * 10^{7} \mathrm{CFU}$, animals of experimental group -2 - the same strain at the dose of $3 * 10^{8}$ CFU, experimental group-3 - a promising culture of L. plantarum Lc-18 at the dose of $3^{*} 10^{7} \mathrm{CFU}$, experimental group -4 - the same culture at the dose of $3 * 10^{8}$ CFU. The suspensions of bacterial cells in saline were injected to animals once orally. After 30, 60 min., $24 \mathrm{hr} ., 7$ and 14 days 3 animals from each group were taken out of the experiment, the samples of their liver, spleen, kidneys, and blood were taken $[3 ; 5]$.

Blood was mixed with a defined part of anticoagulant during the selection; homogenates on the saline were received from parenchymal organs in the ratio of 1:9 (dilution $10^{-1}$ ). To determine the presence and quantity of bacteria that translocated to the blood and parenchymal organs of animals, sowings of tenfold dilution of obtained samples in test tubes with MRS medium were done [10]. Accounting was made according to Mc Credit tables [15], making the recount on the addition of anticoagulant for blood samples.

Housing, feeding, care and all manipulations with laboratory animals were carried out under the European Convention "For the protection of vertebrate animals used for experimental and other scientific purposes" (Strasbourg, 1986) [16] and "General ethical principles of experiments on animals" approved at the first National Congress on Bioethics (Kyiv, 2001) [17]. Experiments were conducted in compliance with the principles of humanity set out in the European Community Directive [18].

Mathematical processing of the results, which are quantified, was made by the methods of variation statistics using Student t-test and correlation coefficients [19].

So, a crucial dependence of bacterial translocation manifestation from introduced strain of bacteria was established. Thus, the bacterial strain Lactobacillus plantarum L5 is characterized by weak capacity for translocation into the blood and parenchymal organs of a macroorganism, while the other investigated microorganism translocation ( $L$. plantarum Lc-18) is intense.

The dependence of the dynamics of lactic acid bacteria translocation from the dose was established: the introduction of bacteria, which are used to check the safety of probiotics $\left(3 * 10^{8}\right.$ $\mathrm{CFU})$ is accompanied by intense translocation, beginning in the first 10 minutes after the introduction and the concentration of cells in the body remains stable for a quite long time ( 7 days); the introduction of a lower dose of bacteria $\left(3 * 10^{7} \mathrm{CFU}\right)$ causes a smoother start of a bacterial translocation and significantly lower concentrations of bacteria in the blood and tissues of parenchymal organs. 